\title{
Turbulence-Flame Interaction on the Early Evolution of Flames in Type la Supernovae
}

\author{
Aaron P. Jackson* \\ Stony Brook University \\ E-mail: Aaron. Jackson@stonybrook.edu
}

\section{Alan C. Calder}

Stony Brook University

\section{Dean M. Townsley}

University of Alabama

\section{David A. Chamulak}

Argonne National Laboratory

\section{Edward F. Brown}

Michigan State University

\section{F. X. Timmes}

\section{Arizona State University}

Type Ia supernovae are bright stellar explosions thought to occur when a runaway thermonuclear reaction incinerates a compact star known as white dwarf (WD). In many models, the explosion begins with a flame born in the turbulent environment near the center of the white dwarf. The effect of turbulence on the evolution of the nascent flame is incompletely understood and is the subject of active research. The range of length scales from the full star $\left(\sim 10^{8} \mathrm{~cm}\right)$ to the laminar flame width $\left(\sim 10^{-5} \mathrm{~cm}\right)$ prevents full-star simulations from resolving the turbulence-flame interaction (TFI) directly. In the single-degenerate paradigm of Type Ia supernovae ( $\mathrm{SNe}$ Ia), the WD experiences $\sim 1000$ year period of convection as the temperature rises to burn carbon. When the nuclear burning timescale exceeds the turnover time for convective eddies, a flame is born in the center of a vigorous convection field $\left(v_{\text {rms }} \sim 400 \mathrm{~km} / \mathrm{s}\right.$ ) extending out to enclose $\sim 70 \%$ of the WD's mass [1]. We present preliminary results from a physically-motivated TFI model inspired by Colin et al. (2000) [2] that utilizes a local, instantaneous measure of the turbulence to enhance the flame speed due to under-resolved TFI. We explore various implementation choices in the TFI model and compare results to previous work. We present two simulations of the early flame evolution in a supernova. One incorporates a TFI model with particular implementation choices, while the other relies only on indirect buoyancy effects [3].

11th Symposium on Nuclei in the Cosmos, NIC XI

July 19-23, 2010

Heidelberg, Germany

\footnotetext{
* Speaker.
} 


\section{Turbulence Flame Interaction Model}

Colin et al. (2000) [2] developed a TFI subgrid scale (SGS) model that constructs a wrinkling factor for the flame based on the strain rate given by the turbulent velocity measured at some scale within the inertial subrange of the assumed Kolmogorov turbulence cascade. A wrinkling factor is computed for both the thickened flame resolved on the computational grid and the real unresolved flame at the density and composition in the degenerate WD. The wrinkling factors are normalized to the limiting behavior where all turbulent motions in the cascade strain the flame front that results in the Damköhler scaling behavior $1+u / s$ where $u$ is the turbulent velocity at the integral scale and $s$ is the laminar flame speed. The enhancement to the thickened flame speed is calculated from the ratio of wrinkling factors for the real and thickened flames.

Two primary implementations of the TFI model were developed and explored. For the first, we choose to normalize the wrinkling factors such that the wrinkling factor for the thin flame always obeys Damköhler scaling. For the second, the wrinkling factors are normalized such that the maximal strain rate reproduces Damköhler scaling. The former implementation is denoted by "D", while the latter is denoted by "K" throughout this presentation. The two implementations are consistent when the Kolmogorov scale $\eta_{k}$ is set to the thin flame width $\delta_{l}^{0}$ (see Figure 1).

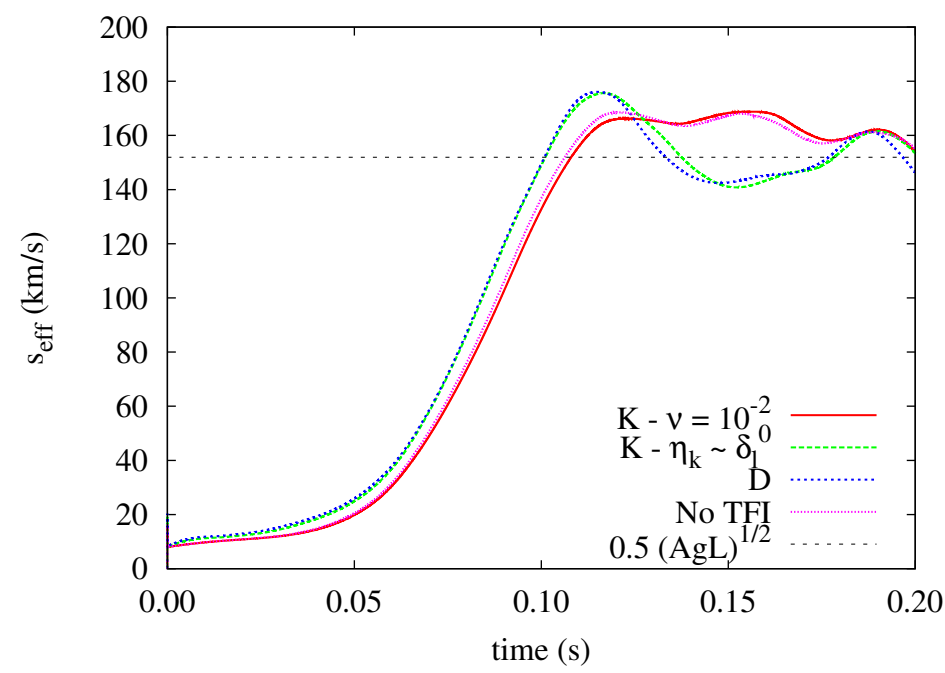

Figure 1: We compare the effective propagation speeds of a flames assigned a constant speed of $9 \mathrm{~km} / \mathrm{s}$ in a channel with constant gravity. For a typical viscosity in a WD (red), implementation "K" produces no enhancement (magenta) due to $\eta_{k} \ll \delta_{l}^{0}$ such that most of the inertial subrange does not wrinkle the flame. By choosing the viscosity in TFI implementation "K" such that $\eta_{k} \sim \delta_{l}^{0}$ (green), all turbulent motions in the inertial subrange wrinkle the flame resulting in Damköhler scaling consistent with implementation "D" (blue).

\section{Methodology}

We use an advection-diffusion-reaction scheme within the Flash code $[4,5]$ to propagate a thickened flame representing the $\mathrm{C}$ deflagration with subsequent stages of nuclear burning. The scheme takes as input a tabulated flame speed [9] and compensates for buoyancy effects of the 


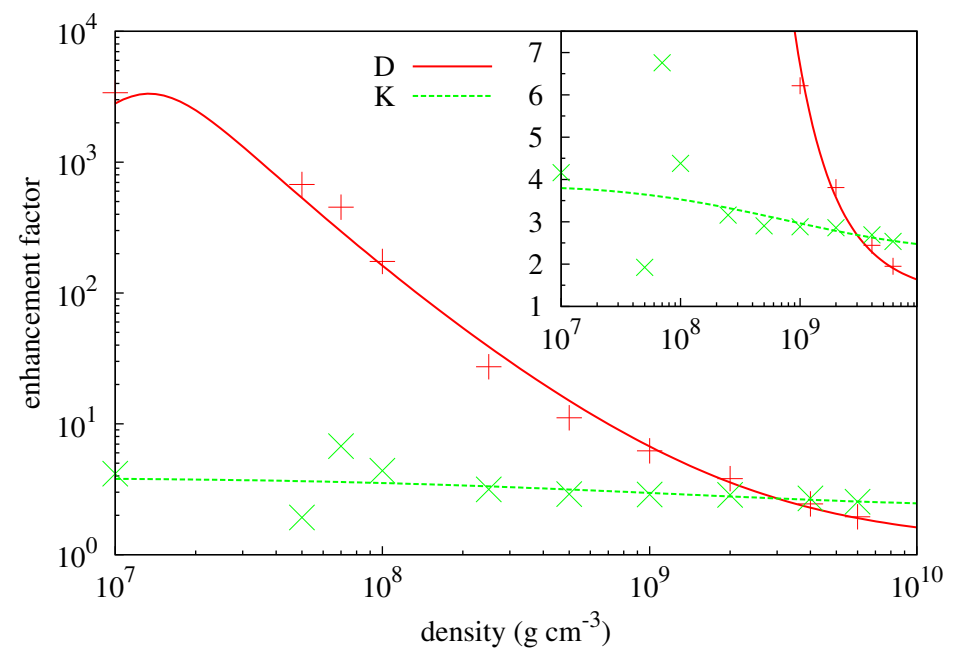

Figure 2: The enhancement factor to the flame speed is calculated with both " $D$ " (red) and "K" (green) TFI model implementations as a function of the fuel density using a turbulent velocity of $400 \mathrm{~km} / \mathrm{s}$. The curves are estimated by fitting laminar flame speeds and widths as a function of density from a 50/50 C-O flame to tabulated values corresponding to the points [9]. The model flame width is assumed to be $16 \mathrm{~km}$, typical of a $4 \mathrm{~km}$ resolution simulation. An inset with the enhancement (linear y-axis) is provided for comparison.
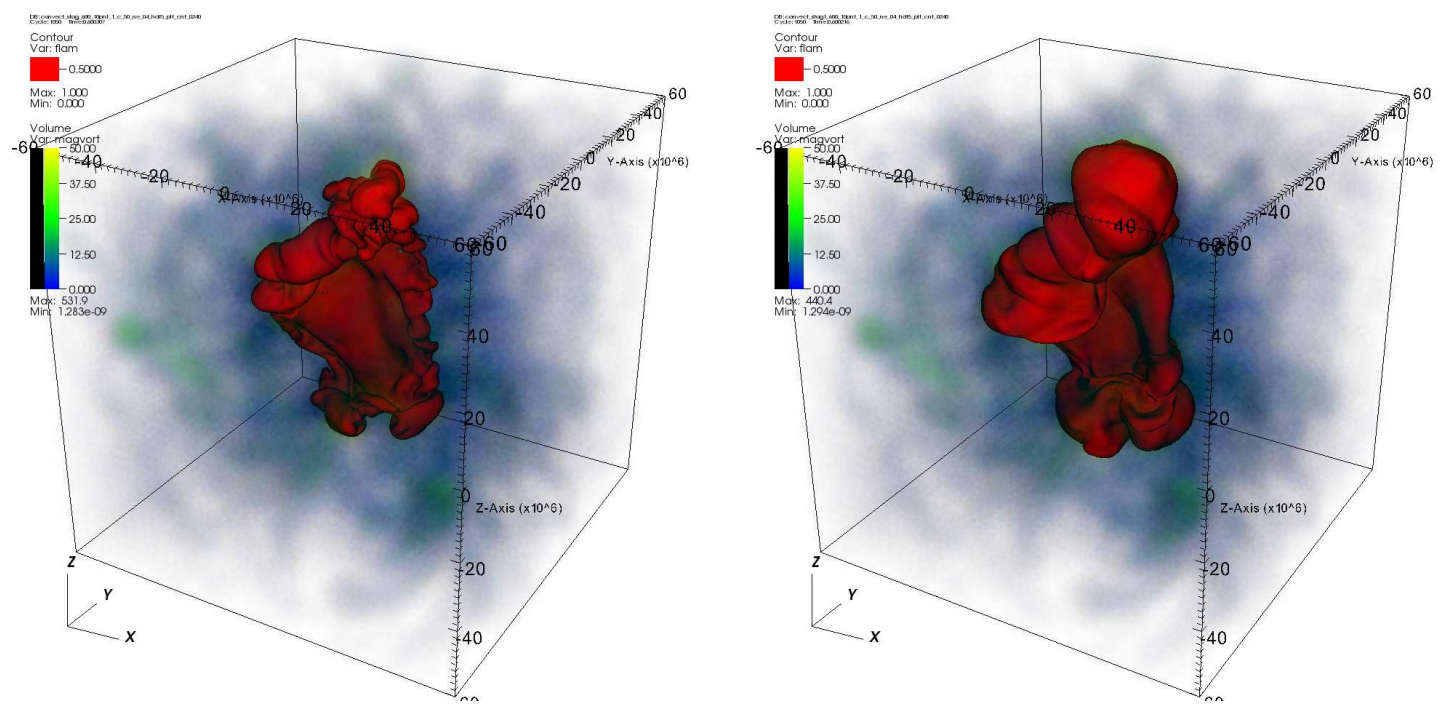

Figure 3: We compare the evolution of the early flame interacting with a turbulent velocity field (blue) in a thermonuclear supernova. Implementation "K" of the TFI model utilizing a local measure of the turbulent velocity (right) is compared to an indirect treatment of the TFI using only buoyancy effects (left). We find that our local treatment of TFI produces a larger enhancement to the flame speed as indicated by a larger volume enclosed by the flame surface.

Rayleigh-Taylor unstable flame front. The energetics and time-scales for the burning are taken from prior calculations. The detonation is propagated by thermally activated reactions. Complete details are in $[6,7,8]$. 


\section{Results}

The expected enhancement from a physically motivated TFI model in Figure 2 demonstrates the importance of including a physically-motivated TFI model to capture the early dynamics of the deflagration phase of SNe Ia (see Figure 3). Figure 4 shows the details of the TFI model impact the expansion of the WD and thus the total Fe-group yield. Schmidt et al. (2006) [11] also developed a complex SGS TFI model that considers SGS turbulent energy production, transport, and destruction. In contrast, Colin et al. (2000) [2] utilize a relatively simple local, instantaneous measure of the turbulent velocity and assume a Kolmogorov turbulence cascade. Implementing different SGS TFI models should highlight the impact of certain assumptions in the construction of these models.

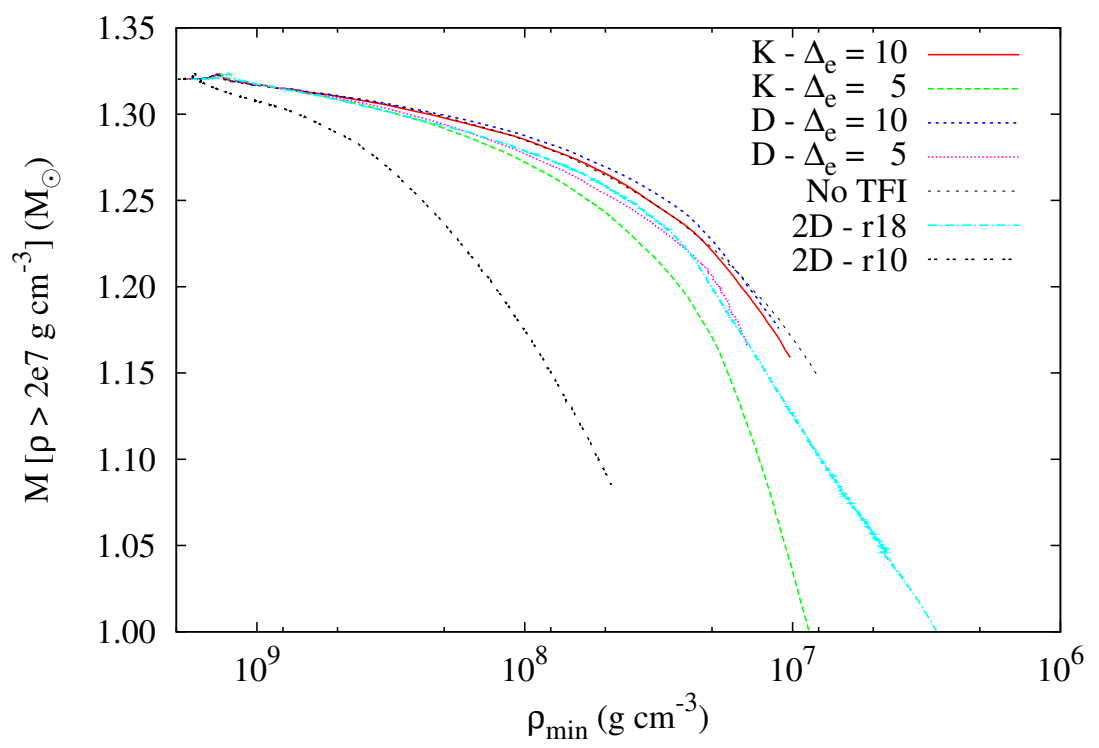

Figure 4: We compare expansion histories by plotting the evolution of mass with density $>2 \times 10^{7} \mathrm{~g} \mathrm{~cm}^{-3}$ as a function of the minimum flame density utilizing different implementations of the TFI model. The mass with density $>2 \times 10^{7} \mathrm{~g} \mathrm{~cm}^{-3}$ provides an estimate of the Fe-group yield when the minimum flame density reaches the deflagration-to-detonation density. In order to estimate the enhancement to the flame speed, we measure the turbulent velocity locally near the grid scale. Curves with different $\Delta_{e}$ indicate the filter size in units of grid cell widths for the measurement operation. Shown for comparison are curves from our 2D study [10] resulting in the highest yield (r18) and lowest yield ( $(\mathrm{r} 10)$ indicating that the green curve shows a similar expansion history to that calculated in $2 \mathrm{D}$.

\section{Acknowledgments}

This work was supported by the Department of Energy through grants DE-FG02-07ER41516, DE-FG02-08ER41570, and DE-FG02-08ER41565, and by NASA through grant NNX09AD19G. A.C.C. acknowledges support from the Department of Energy under grant DE-FG02-87ER40317. D.M.T. received support from the Bart J. Bok fellowship at the University of Arizona for part of this work. This work was supported in part by the US Department of Energy, Office of Nuclear 
Physics, under contract DE-AC02-06CH11357. The authors gratefully acknowledge the use of NSE and weak reaction tables developed by Ivo Seitenzahl. The authors also acknowledge the hospitality of the KITP, which is supported by NSF grant PHY05-51164, during the programs "Accretion and Explosion: the Astrophysics of Degenerate Stars" and "Stellar Death and Supernovae." The software used in this work was in part developed by the DOE-supported ASC/Alliances Center for Astrophysical Thermonuclear Flashes at the University of Chicago. This research utilized resources at the New York Center for Computational Sciences at Stony Brook University/Brookhaven National Laboratory which is supported by the U.S. Department of Energy under contract no. DEAC02-98CH10886 and by the State of New York.

\section{References}

[1] M. Zingale, A. S. Almgren, J. B. Bell, A. Nonaka, and S. E. Woosley. Low Mach Number Modeling of Type IA Supernovae. IV. White Dwarf Convection. Astrophysical Journal, 704:196-210, October 2009.

[2] O. Colin, F. Ducros, D. Veynante, and T. Poinsot. A thickened flame model for large eddy simulations of turbulent premixed combustion. Physics of Fluids, 12:1843-1863, July 2000.

[3] A. M. Khokhlov. Delayed detonation model for type Ia supernovae. Astronomy and Astrophysics, 245:114-128, 1991

[4] B. Fryxell, K. Olson, P. Ricker, F. X. Timmes, M. Zingale, D. Q. Lamb, P. MacNeice, R. Rosner, J. W. Truran, and H. Tufo. FLASH: An adaptive mesh hydrodynamics code for modeling astrophysical thermonuclear flashes. Astrophysical Journal Supplement, 131:273-334, 2000.

[5] A. C. Calder, B. Fryxell, T. Plewa, R. Rosner, L. J. Dursi, V. G. Weirs, T. Dupont, H. F. Robey, J. O. Kane, B. A. Remington, R. P. Drake, G. Dimonte, M. Zingale, F. X. Timmes, K. Olson, P. Ricker, P. MacNeice, and H. M. Tufo. On validating an astrophysical simulation code. Astrophysical Journal Supplement, 143:201-229, 2002.

[6] A. C. Calder, D. M. Townsley, I. R. Seitenzahl, F. Peng, O. E. B. Messer, N. Vladimirova, E. F. Brown, J. W. Truran, and D. Q. Lamb. Capturing the Fire: Flame Energetics and Neutronization for Type Ia Supernova Simulations. Astrophysical Journal, 656:313-332, February 2007.

[7] D. M. Townsley, A. C. Calder, S. M. Asida, I. R. Seitenzahl, F. Peng, N. Vladimirova, D. Q. Lamb, and J. W. Truran. Flame Evolution During Type Ia Supernovae and the Deflagration Phase in the Gravitationally Confined Detonation Scenario. Astrophysical Journal, 668:1118-1131, October 2007.

[8] D. M. Townsley, A. P. Jackson, A. C. Calder, D. A. Chamulak, E. F. Brown, and F. X. Timmes. Evaluating Systematic Dependencies of Type Ia Supernovae: The Influence of Progenitor ${ }^{22} \mathrm{Ne}$ Content on Dynamics. Astrophysical Journal, 701:1582-1604, August 2009.

[9] D. A. Chamulak, E. F. Brown, and F. X. Timmes. The Laminar Flame Speedup by ${ }^{22} \mathrm{Ne}$ Enrichment in White Dwarf Supernovae. Astrophysical Journal Letters, 655:L93, February 2007.

[10] A. P. Jackson, A. C. Calder, D. M. Townsley, D. A. Chamulak, E. F. Brown, and F. X. Timmes. Evaluating Systematic Dependencies of Type Ia Supernovae: The Influence of Deflagration to Detonation Density. Astrophysical Journal, 720:99-113, September 2010.

[11] W. Schmidt, J. C. Niemeyer, W. Hillebrandt, and F. K. Röpke. A localised subgrid scale model for fluid dynamical simulations in astrophysics. II. Application to type Ia supernovae. Astronomy and Astrophysics, 450:283-294, April 2006. 